\title{
Omega-3 fatty acid deficiency disrupts endocytosis, neuritogenesis, and mitochondrial protein pathways in the mouse hippocampus
}

\author{
Jane A. English ${ }^{1,2 *}$, Akiko Harauma ${ }^{3}$, Melanie Föcking ${ }^{1}$, Kieran Wynne ${ }^{2}$, Caitriona Scaife ${ }^{2}$, \\ Gerard Cagney ${ }^{2}$, Toru Moriguchi ${ }^{3}$ and David R. Cotter ${ }^{1}$
}

\author{
${ }^{1}$ Department of Psychiatry, Royal College of Surgeons in Ireland, ERC Beaumont Hospital, Dublin, Ireland \\ 2 Proteome Research Centre, School of Medicine and Medical Science, UCD Conway Institute of Biomolecular and Biomedical Research, University College of \\ Dublin, Dublin, Ireland \\ ${ }^{3}$ Department of Food and Life Science, Azabu University, Sagamihara, Japan
}

Edited by:

Yannis Karamanos, Université

d'Artois, France

Reviewed by:

Ka Wan Li, VU University,

Netherlands

Hitoshi Hashimoto, Osaka

University, Japan

*Correspondence:

Jane A. English, Department of

Psychiatry, Royal College of

Surgeons in Ireland, ERC Beaumont

Hospital, Beaumont, Dublin 9,

Ireland

e-mail: janeenglish@rcsi.ie

Omega-3 fatty acid (n-3 FA) deficiency is an environmental risk factor for schizophrenia, yet characterization of the consequences of deficiency at the protein level in the brain is limited. We aimed to identify the protein pathways disrupted as a consequence of chronic $n-3$ deficiency in the hippocampus of mice. Fatty acid analysis of the hippocampus following chronic dietary deficiency revealed a 3-fold decrease $(p<0.001)$ in n-3 FA levels. Label free LC-MS/MS analysis identified and profiled 1008 proteins, of which 114 were observed to be differentially expressed between n-3 deficient and control groups ( $n=8$ per group). The cellular processes that were most implicated were neuritogenesis, endocytosis, and exocytosis, while specific protein pathways that were most significantly dysregulated were mitochondrial dysfunction and clathrin mediated endocytosis (CME). In order to characterize whether these processes and pathways are ones influenced by antipsychotic medication, we used LC-MS/MS to test the differential expression of these 114 proteins in the hippocampus of mice chronically treated with the antipsychotic agent haloperidol. We observed 23 of the 114 proteins to be differentially expressed, 17 of which were altered in the opposite direction to that observed following n-3 deficiency. Overall, our findings point to disturbed synaptic function, neuritogenesis, and mitochondrial function as a consequence of dietary deficiency in n-3 FA. This study greatly aids our understanding of the molecular mechanism by which n-3 deficiency impairs normal brain function, and provides clues as to how n-3 FA exert their therapeutic effect in early psychosis.

Keywords: Omega-3, proteomics, neuritogenesis, mitochondrial dysfunction, clathrin mediated endocytosis, schizophrenia

\section{INTRODUCTION}

Important new evidence indicates that dietary omega-3 fatty acid's (n-3 FA) significantly reduces the risk of progression to psychosis in adolescences with at-risk mental state (ARMS) (Amminger et al., 2010). Other studies have reported reduced n-3 FA in red blood cell membranes, platelets, and in postmortem brain of patients with schizophrenia (McNamara, 2009), and there is now strong evidence to suggest that n-3 FA deficiency is a preventable risk factor for schizophrenia (McNamara, 2011). Preclinical rodent studies have demonstrated that supplementation with n-3 FA can reverse stress induced behavioral and cognitive abnormalities (Ferraz et al., 2011; Feng et al., 2012), and even protect against MK-801 induced neurotoxicity in the prefrontal cortex (Ozyurt et al., 2007). More recently, Bondi and colleagues have shown that $\mathrm{n}-3$ FA deficiency across consecutive generations in a rat model caused impaired cognitive and motivated behavior in adolescent rats (Bondi et al., 2013). The consequences of dietary n-3 deficiency on molecular pathways within the brain are relatively unexplored at the level of the proteome.

Polyunsaturated n-3 FA, particularly docosahexaenoic acid (DHA), are selectively concentrated within the phospholipid bilayer of cell membrane structures in the brain (e.g., synaptic membranes, vesicles, dendritic membranes, and mitochondria). If unavailable, these essential polyunsaturated FA are replaced by non-essential fatty acids, thus alterations in the membrane microenvironment and fluidity can occur, disrupting neuronal membrane dynamics, as well as receptor, transporter, and neurotransmitter function (Horrobin, 1998). Animal models have previously shown that DHA can modulate gene expression (Kitajka et al., 2002) including enhanced dopamine receptor gene expression following maternal deficiency (Kuperstein et al., 2005). In addition, DHA has been shown to enhance neuritogenesis (Kawakita et al., 2006; Beltz et al., 2007; He et al., 2009), and improve mitochondrial function in in vivo and ex vivo animal models of neurodegenerative disease (Eckert et al., 2013). In order 
to fully understand the sub-cellular cellular processes that are altered as a consequence of n-3 FA deficiency, we used a discovery based approach to profile differential protein expression changes within the hippocampus of mice following n-3 FA prenatal and trans-generational deficiency. In addition, given our previous findings implicating the cellular trafficking process of clathrin mediated endocytosis (CME) in schizophrenia (Föcking et al., 2011) and evidence implicating n-3 FA in CME in cell culture (Ben Gedalya et al., 2009), we hypothesized that n-3 deficiency influences CME processes in the brain. Therefore, the aim of this study was twofold; (1) To identify protein pathways disrupted as a consequence of n-3 FA deficiency, and (2) To identify CME related proteins that are disrupted as a consequence of n-3 FA deficiency. Using label free LC-MS/MS quantitative proteomics, our investigation focused on the hippocampus from mice chronically deficient in dietary n-3 fatty acids.

\section{MATERIALS AND METHODS EXPERIMENTAL DIETS AND ANIMALS}

Mice were fed either an n-3 fatty acid deficient (n-3 Def) or $\mathrm{n}-3$ adequate ( $\mathrm{n}-3 \mathrm{Adq}$ ) diet for two generations, as previously described (Harauma and Moriguchi, 2011). The experimental diets were based on the AIN-93G diet recommendations for rodents (Reeves et al., 1993), with modifications of the fat source (flaxseed oil) to achieve the low n-3 fatty acid level required (Table 1). The fat content in both diets was $5 \%(\mathrm{w} / \mathrm{w})$ diet, and there was no difference in the total n- 6 fatty acids between the two diets. The diet was custom prepared at Oriental Yeast, Chiba, Japan.

Female CD-1 (ICR) mice were obtained at 3 weeks of age from Charles River Japan, Inc. and fed an n-3 Def or n-3 Adq diet. Animals were maintained within the animal facility under conventional conditions, as previously described (Harauma and Moriguchi, 2011). At 8 weeks of age, they were mated with 9-week-old males of the same strain. Their litters (1st generation) were culled to ten pups and the pups were weaned onto the same diet as their Dams. The male and female offspring (2nd generation) were maintained on each diet. When they were 10 weeks old, brain was collected, and the hippocampus was dissected from the whole brain and frozen at $-80^{\circ} \mathrm{C}$ prior fatty acid analysis. This experimental protocol was approved by the audit committee of Azabu University Animal Experiment, according the guidelines of the Animal Experiment in Azabu University.

To confirm n-3 deficiency in brain tissue, lipid extraction and gas chromatography was carried out as previously described in (Harauma and Moriguchi, 2011), according to the method of Lepage and Roy (1986). Internal standards were used to calculate tissue fatty acid concentration. All data were expressed as the mean \pm Standard Error of the Mean (SEM). The fatty acid composition in the hippocampus tissues were evaluated using the Tukey's test after One-Way ANOVA (Statistica, Statsoft Japan, Tokyo, Japan) at $P<0.05$.

\section{SAMPLE PREPARATION AND LABEL FREE QUANTIFICATION USING LC-MS/MS ANALYSIS}

For label free LC-MS/MS analysis, the hippocampus of 16 n-3 Def and 16 n-3 Adq samples per dietary group were assessed. Prior to
Table 1 | Composition of experimental diets.

\begin{tabular}{|c|c|c|}
\hline & \multicolumn{2}{|c|}{ Composition (g/100g) } \\
\hline & n-3 Adq. & n-3 Def. \\
\hline Casein, vitamin free & 20 & 20 \\
\hline Carbohydrate: & 65 & 65 \\
\hline Cornstarch & 10 & 10 \\
\hline$\alpha$-Cornstarch & 15 & 15 \\
\hline Sucrose & 10 & 10 \\
\hline Glucose & 20 & 20 \\
\hline Dextrose & 5 & 5 \\
\hline Maltose-dextrin & 5 & 5 \\
\hline Cellulose & 5 & 5 \\
\hline Mineral-salt mix & 3.5 & 3.5 \\
\hline Vitamin mix & 1 & 1 \\
\hline L-Cystine & 0.3 & 0.3 \\
\hline Choline bitartrate & 0.25 & 0.25 \\
\hline $\mathrm{TBHO}$ & 0.001 & 0.001 \\
\hline Fat: & 5 & 5 \\
\hline Hydrogenated coconut oil & 3.875 & 4.05 \\
\hline Saffower oil & 0.885 & 0.95 \\
\hline Flaxseed oil & 0.24 & none \\
\hline \multicolumn{3}{|l|}{ Fatty acid composition ${ }^{a}$} \\
\hline Saturates & 75.1 & 78.1 \\
\hline Monounsaturates & 5.4 & 4.7 \\
\hline $18: 2 n-6$ & 14.6 & 14.6 \\
\hline $18: 3 n-3$ & 2.53 & 0.25 \\
\hline$n-6 / n-3$ & 5.8 & 58.5 \\
\hline
\end{tabular}

The two experimental diets, an n-3 fatty acid dequate diet (n-3 Adq) and an n-3 fatty acid deficient diet (n-3 Def), were based on the AIN-93 formulation with several modifications to obtain the extremely low basal level of n-3 fatty acid required in this study.

a The 20:4n-6, 20:5n-3 and 22:6n-3 fatty acids were less than 0.01\%, i.e., not detected (nd).

analysis, these 16 samples were sub-pooled according to gender $(4$ male and 4 female per dietary group) so that $8 \times \mathrm{n}-3$ Def and $8 \times$ n-3 Adq LC-MS/MS runs were performed. Following solubilisation of samples by sonication in triethylammonium bicarbonate (TEAB; Sigma), $50 \mu \mathrm{g}$ of protein from each of the $8 \mathrm{n}-3$ Adq and $8 \mathrm{n}-3$ Def samples was denatured for $10 \mathrm{~min}$ at $80^{\circ} \mathrm{C}$ in the presence of $1 \%$ RapiGest (Waters). Samples were reduced with TCEP $(50 \mathrm{mM})$ for $60 \mathrm{~min}$ at $60^{\circ} \mathrm{C}$, followed by alkylation with Iodoacetamide $(200 \mathrm{mM})$ for $30 \mathrm{~min}$ in the dark. Digestion with trypsin (Promega; $1 \mu \mathrm{g} / \mu \mathrm{l}$ ) was carried out overnight at $37^{\circ} \mathrm{C}$, and Tryptic peptides were purified using ZipTips (Millipore) according to the manufacturer's instructions. Samples were resuspended in Solution A (HPLC grade water, 2\% acetonitrile, 0.5\% acetic acid) where by $1 \mu \mathrm{g}$ of protein digest $(5 \mu \mathrm{l})$ was injected per LC$\mathrm{MS} / \mathrm{MS}$ run. Each of the 16 samples were run in triplicate, on a Thermo Scientific LTQ ORBITRAP XL mass spectrometer connected to an Dionex Ultimate 3000 (RSLCnano) chromatography system. Each sample was loaded onto Biobasic Picotip Emitter (120 mm length, $75 \mu \mathrm{m}$ ID) packed in house with Reprocil Pur C18 $(1.9 \mu \mathrm{m})$ reverse phase media column, and was separated by an increasing acetonitrile gradient, using a 56 min reverse phase 
gradient at a flow rate of $250 \mathrm{~nL} / \mathrm{min}$. From 0 to $7 \mathrm{~min} 5 \mu \mathrm{l}(1 \mu \mathrm{g})$ of sample was injected and loaded onto the column at a flow rate of $1.5 \mu \mathrm{l} / \mathrm{min}$. At $7 \mathrm{~min}$ the mass spectrometer started to acquire data, the chromatography gradient continued, 7-44 min from 5 to $35 \%$ Solution B (acetonitrile containing 3\% HPLC grade water and $0.5 \%$ acetic acid) at $250 \mathrm{nl} / \mathrm{min}, 44-50 \mathrm{~min}$ from 35 to $90 \%$ Solution B at $250 \mathrm{nl} / \mathrm{min}, 50-55 \mathrm{~min}$ at $90 \%$ Solution B at $250 \mathrm{nl} / \mathrm{min}$ increasing to $900 \mathrm{nl} / \mathrm{min}, 55-56 \mathrm{~min}$ at $90 \%$ Solution B to $2 \%$ Solution B at $900 \mathrm{nl} / \mathrm{min}$ decreasing to $250 \mathrm{nl} / \mathrm{min}$. The mass spectrometer was operated in positive ion mode with a capillary temperature of $200^{\circ} \mathrm{C}$, a capillary voltage of $45 \mathrm{~V}$, a tube lens voltage of $100 \mathrm{~V}$ and with a potential of $1800 \mathrm{~V}$ applied to the frit. Survey full-scan MS spectra (300-2000 Da) were acquired in the Orbitrap with a resolution of 60,000, whereby the FTMS maximum injection time was $700 \mathrm{~ms}$, the Ion trap injection time was $100 \mathrm{~ms}$, and the 7 most intense ions from the preview scan were selected for MS/MS analysis.

\section{DATA ANALYSIS}

Label-free quantification (LFQ) was performed with Max Quant (V 1.3.0.2) as previously described (Hubner et al., 2010). Methionine oxidations and acetylation of protein $\mathrm{N}$-termini were specified as variable modifications, while carbamidomethylation was specified as a fixed modification, and 2 miscleavages were allowed. Protein and peptide FDR's were set to 0.01 . Only proteins with at least two peptides (one uniquely assignable to the protein) were considered as reliably identified. LFQ intensity values were used for protein quantification between groups. Only unique and razor peptides were considered for quantification with a minimum ratio count of. Statistical analyses was performed in Perseus (V 1.3.0.4), whereby the data was $\log 2$ transformed, missing values were replaced by values form the normal distribution, and column normalization was performed by subtracting the median. Student's $t$-test was applied to identify proteins differentially expressed between groups at a 5\% threshold, and a permutationbased FDR was applied at a 5\% threshold. Ingenuity Pathway Analysis (IPA; www.ingeunity.com) was performed on all statistically significant proteins. The IPA $p$-value was calculated using the right-tailed Fisher's Exact Test and multiple hypothesis correction was based on the Benjamini-Hochberg $(\mathrm{B}-\mathrm{H})$ approach at $1 \%$ FDR threshold.

\section{HALOPERIDOL TREATED MICE}

In order to assess the effects of psychotropic medication on the expression of the specific candidate proteins identified as altered as a consequence of n-3 deficiency, hippocampal tissue was harvested from C57BL6 mice chronically treated with haloperidol (28 days), as previously described (Föcking et al., 2011). Ethical approval (application no.175) was granted by the Royal College of Surgeons in Ireland Research Ethics Committee. The tissue was processed and analyzed by LC-MS/MS as above.

\section{RESULTS}

\section{ANIMALS AND FATTY ACID ANALYSIS OF BRAIN TISSUE}

The diets produced no significant differences in body weight gain between dietary groups. In the fatty acid composition analysis of the hippocampus, total n-3 fatty acids were significantly decreased in the n-3 Def group $(p<0.001)$, in each gender (Table 2). This decrease is mostly attributed the reduced levels of docosahexaenoic acid (DHA; 22:6n-3; Table 2). Saturated and monounsaturated fatty acid were approximately the same level. It is noteworthy that the levels of omega- 6 fatty acids (n-6 FA) such $22: 4 n-6$ and 22:5n-6 are considerably increased in the n3 Def mice. It is well-documented that in the absence of n-3 fatty acids, the brain replaces them with other long chain fatty acids such as 22:4n-6 and 22:5n-6, and these findings are in keeping with previous fatty acid composition analyses from n-3 FA deficient mice (Moriguchi et al., 2000; Harauma and Moriguchi, 2011).

\section{LABEL FREE LC-MS/MS QUANTITATIVE ANALYSIS}

For label free LC-MS/MS, we assessed the hippocampus of 8 samples per dietary group, including 4 male (sub-pool of 8 mice) and 4 female (sub-pool of 8 mice) in each group. To account for technical variability and reduce the risk of detecting false positives, samples were run in triplicate on the Thermo LTQ Orbitrap. The average MS1 peak width was $30 \mathrm{~s}$, and the scan time ranged between 1 and $2 \mathrm{~s}$, whereby an average of 15 data points were acquired for quantitation (Supplementary Figure S1). Using Max Quant we identified and quantified 1008 proteins for differential expression between the 8 n-3 Def and 8 n-3 Adq samples of the hippocampus. Expression profiling identified 114 proteins as significantly differentially expressed $(p<0.05)$ between the dietary groups (student's $t$ test). Of these, 61 proteins were significantly increased, and 53 proteins were significantly decreased in expression. Six proteins were significant following permutation-based FDR at 5\% threshold. Supplementary Table $\mathbf{S} 1$ lists the proteins significantly altered as a consequence of dietary n-3 deficiency in the hippocampus, and proteins that remained significant following FDR are marked with an asterisks.

\section{PATHWAY ANALYSIS}

Using IPA we identified cellular function and maintenance as the most significant function disrupted in n-3 FA deficiency (Supplementary Figure S2, Supplementary Table S2). The subcategories within this process included endocytosis (12 proteins), neuritogenesis ( 15 proteins), and exocytosis (6 proteins) functions as detailed in Table 3. In addition, IPA reported the top canonical pathways altered as a consequence of $n-3$ deficiency (Figure 1, Table 4). These were mitochondrial dysfunction (10 proteins), CME (10 proteins), and 14-3-3 signaling (6 proteins) pathways. Canonical pathways within the IPA knowledge have a defined number of molecules, and a ratio to the total number of molecules in the pathway is reported, as well as a $\mathrm{B}-\mathrm{H}$ p-value. IPA identified 49 of the 114 proteins as previously implicated in neurological disease at the mRNA level (Supplementary Table S1).

\section{CANDIDATE PROTEIN CHANGES IN HALOPERIDOL TREATED MICE}

We examined the hippocampal protein profile from mice chronically treated with haloperidol for the 114 candidate proteins identified to be differentially expressed following n-3 deficiency. 
Table 2 | Fatty acid composition of the hippocampus.

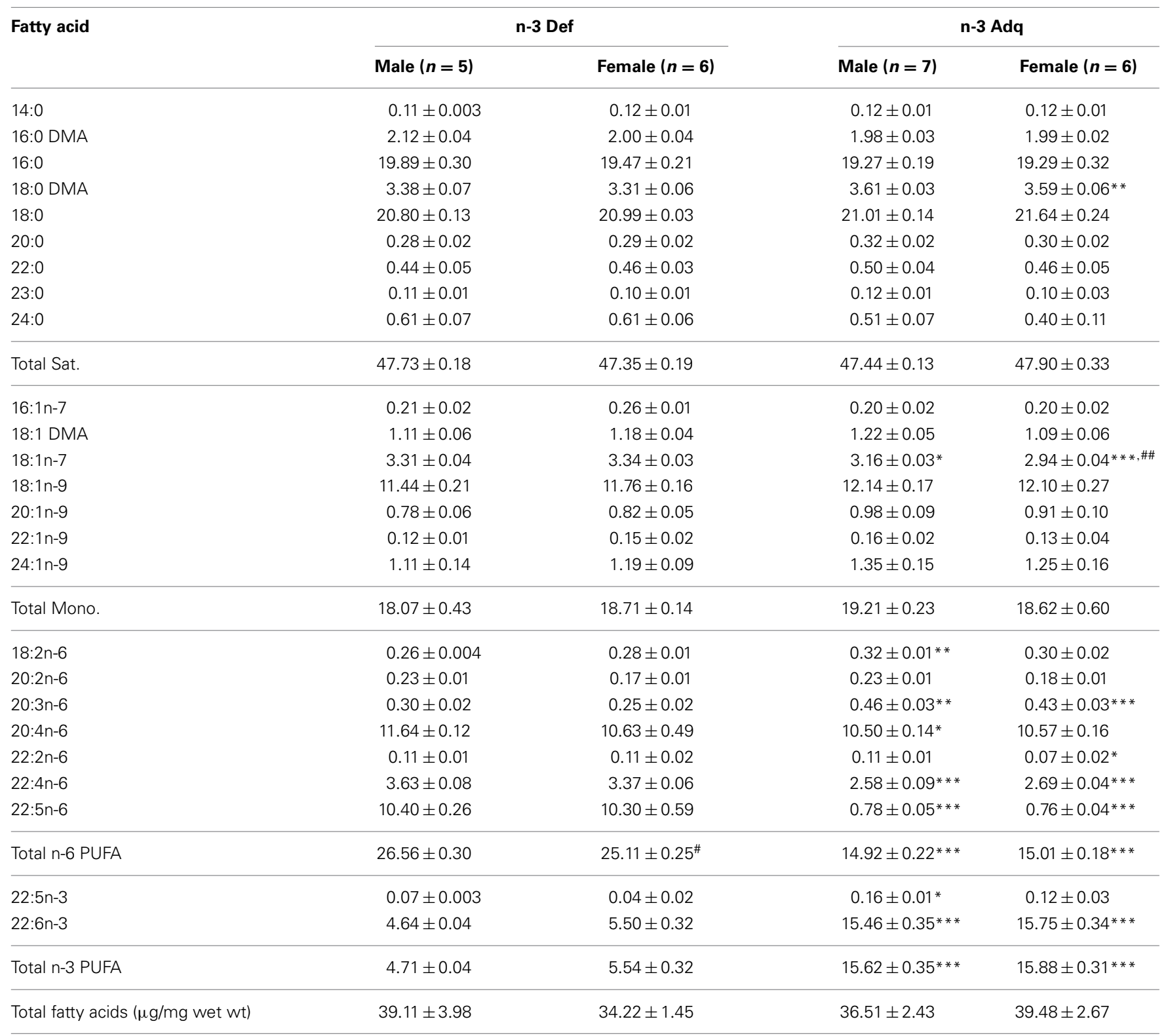

Fatty acid methyl esters from 10:0 to 24:1n-9 were analyzed. 10:0, 12:0, 12:1, 14:1, 18:3n-6, 18:3n-3, 20:3n-3, and 20:5n-3 were not detected (nd, i.e., < 0.01\%). Each parameter is presented as the mean \pm S.E.M. ${ }^{*} P<0.05,{ }^{*} P<0.01,{ }^{* *} P<0.001$ : compared between $n-3$ Def and $n-3$ Adq diet group in each gender. ${ }^{\#} P<0.05, \# P<0.01$ : compared between male and female in each diets (One-Way ANOVA and Tukey test.).

Table 3 | The cellular function and maintenance categories disrupted as a consequence of $n-3$ FA deficiency in order of significance.

\begin{tabular}{|c|c|c|c|}
\hline $\begin{array}{l}\text { Cellular } \\
\text { function } \\
\text { and } \\
\text { maintenance }\end{array}$ & $p$-Value & Molecules & $\begin{array}{l}\text { No. } \\
\text { Molecules }\end{array}$ \\
\hline Endocytosis & 1.79E-04 & APOE,ATP5B,CAP1,CLTC,DNM1L,GRB2,HSPA8,MYO5A,PIP5K1C,PPP3CB,RHOB,SH3GL2 & 12 \\
\hline Neuritogenesis & 4.05E-04 & ABI2,APOE,CTNNA2,DNM1L,GJA1,MYO5A,PIP5K1C,RAB3A,RALA,RHOB,RTN3,RTN4,SEPT11,STMN1,VAPA & 15 \\
\hline Exocytosis & 3.89E-02 & DNM1L,MYO5A,PIP5K1C,RAB3A,RALA,SRCIN1 & 6 \\
\hline
\end{tabular}

These sub-categories include endocytosis, neuritogenesis, and exocytosis functions. 


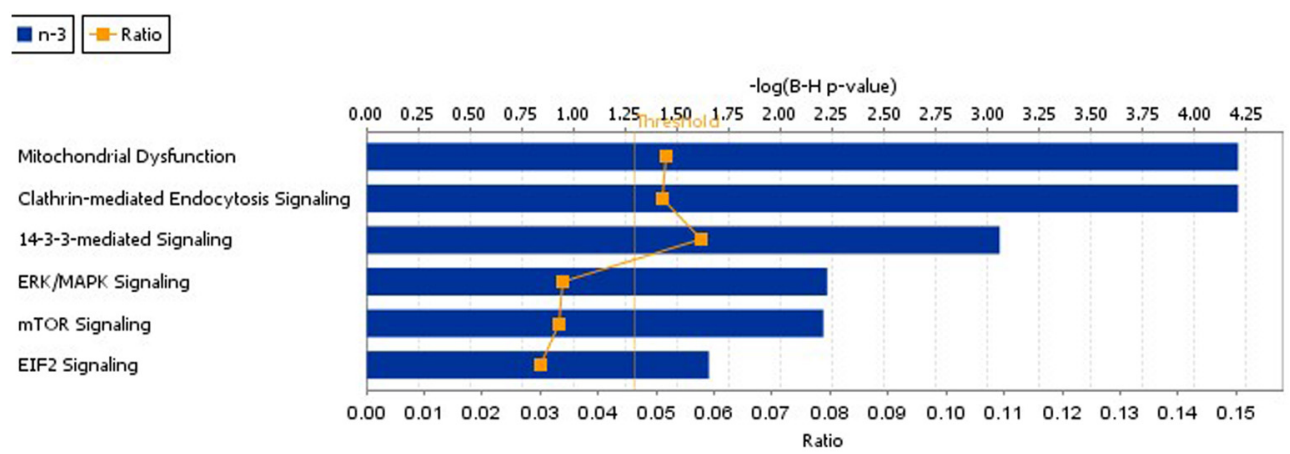

FIGURE 1 | The top IPA canonical pathways disrupted as a consequence of $\mathbf{n}-\mathbf{3}$ FA deficiency. These pathways included mitochondrial dysfunction, CME, 14-3-3 mediated signaling, ERK,/MAPK signaling, mTor, and EIF2 signaling.

Table 4 | The top IPA canonical pathways disrupted as a consequence of $\mathbf{n}-3$ FA deficiency.

\begin{tabular}{|c|c|c|c|}
\hline Ingenuity canonical pathways & $\begin{array}{l}-\log (B-H \\
p \text {-value })\end{array}$ & Ratio & Molecules \\
\hline Mitochondrial dysfunction & 4.21E00 & $10 / 174(0.057)$ & $\begin{array}{l}\text { PDHA1,NDUFV1,ATP5B,ATP5A1,OGDH, UOCRC1, } \\
\text { UOCRC10, NDUFA13,COX4l1,NDUFA8 }\end{array}$ \\
\hline Clathrin-mediated endocytosis & 4.21E00 & 10/196 (0.051) & HSPA8,APOE,PPP3CB,ARPC5L,GRB2,PIP5K1C,CLTC,SH3GL2,DNM1L,UBC \\
\hline 14-3-3-mediated Signaling & 3.06E00 & $7 / 121(0.058)$ & YWHAQ,TUBA1A, YWHAE,GRB2,YWHAZ,TUBA4A,PRKCE \\
\hline ERK/MAPK signaling & 2.23E00 & $7 / 206(0.034)$ & YWHAQ,GRB2,PPP2R4,YWHAZ,PRKAR2A,PRKACA, PRKCE \\
\hline mTOR signaling & $2.21 \mathrm{E} 00$ & $7 / 210(0.033)$ & RPS4Y1,RPS3A,RHOB,PPP2R4,RPS18,PRKCE,RPS25 \\
\hline EIF2 signaling & 1.65E00 & $6 / 200(0.03)$ & RPS4Y1,RPS3A, GRB2,RPS18,RPS25, RPLP0 \\
\hline
\end{tabular}

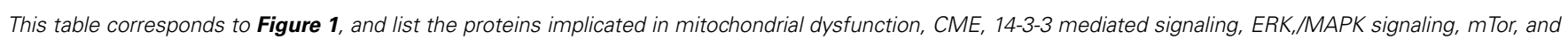
EIF2 signaling.

We used the same LC-MS/MS platform described above. Twentythree of the 114 n-3 candidate proteins were identified as differentially expressed in the haloperidol treated animals and of these, 17 of which were significantly altered in the opposite direction to that observed in n-3 deficiency (Supplementary Table S1).

\section{DISCUSSION}

We used label free LC-MS/MS to study the consequences of n-3 FA deficiency on protein expression in the hippocampus of mice. We profiled 1008 proteins for differential expression, and identified 114 proteins as significantly altered between n-3 deficient and control groups. The major biological processes disturbed by n-3 deficiency included endocytosis, neuritogenesis, and exocytosis (Table 3), and the major signaling pathways altered were mitochondrial dysfunction, CME, 14-3-3 signaling (Table 4). Synaptic function was thus significantly implicated as a biological process (endocytosis and exocytosis) and pathway (CME), followed by disturbances in neuritogenesis, mitochondrial dysfunction, and 14-3-3 signaling. As these functions have previously been reported to be abnormal in schizophrenia (Harrison and Weinberger, 2005; English et al., 2011, 2012; Kim et al., 2012; Manji et al., 2012), our data is consistent with the view that n3 deficiency is an environmental risk factor for schizophrenia (McNamara, 2011).

Synaptic function was significantly disturbed with alternations in both endocytosis (12 proteins) and exocytosis (6 proteins) functions in the hippocampus of n-3 FA deficient mice. More specifically, and in keeping with our hypothesis, the CME protein pathway was implicated, with 6 proteins decreased ( $\downarrow$ GRB2, $\downarrow$ PIP5K1C, $\downarrow$ APOE, $\downarrow$ DNM1L, $\downarrow$ PPP3CB, $\downarrow$ ARPC5L), and 4 increased ( $\uparrow$ CLTC, $\uparrow$ HSPA8, $\uparrow \mathrm{UBC}$, $\uparrow$ SH3GL2) in expression. CME is the best-characterized endocytotic pathway for cellular membrane and protein trafficking, and post-mortem studies implicate $\mathrm{CME}$ as one a potential core pathophysiological processes in schizophrenia (Schubert et al., 2012). Two of the above CME proteins, CLTC (Allen et al., 2008) and HSPA8 (Föcking et al., 2011), have previously been implicated in schizophrenia. In keeping with our data, Ben Gedalya and colleagues have previously shown that n-3 FA augmentation are capable of enhancing CME in neuronal cultured cells (Ben Gedalya et al., 2009). Other endocytosis ( $\uparrow$ RHOB, $\uparrow$ MYO5A, $\uparrow$ ATP5B, $\downarrow$ CAP1), and exocytosis (RAB3A, SRCIN1, RALA) proteins were also significantly altered, along with SNARE protein SNAP 47 ( $\uparrow 2.1$ fold). In addition, the post synaptic density (PSD) protein SHANK3 was significantly increased ( $\uparrow 1.68$ fold) in expression. SHANK3 is a major susceptibility gene in schizophrenia (Gauthier et al., 2010) and we have recently observed decreased SHANK3 expression in the PSD in schizophrenia (Föcking et al., 2012). As disturbed CME and synaptic processes are core features in the pathophysiology of psychiatric disorders, this study provides further evidence that n-3 FA deficiency is a risk factor for schizophrenia. 
Neuritogenesis was the second most significant function implicated following n-3 FA deficiency with 6 proteins significantly decreased ( $\downarrow$ SEPT11, $\downarrow$ PIP5K1C, $\downarrow$ APOE, $\downarrow$ VAPA, $\downarrow$ RALA, $\downarrow$ DNM1L), and 9 increased ( $\uparrow A B 3 A, \uparrow S T M N 1$, $\uparrow \mathrm{GJA1}, \uparrow A B I 2, \uparrow \mathrm{RTN} 3, \uparrow \mathrm{RTN} 4, \uparrow \mathrm{CTNNA2}, \uparrow \mathrm{MYO} 5 \mathrm{~A}$, $\uparrow \mathrm{RHOB})$ in expression. Seven neuritogenesis proteins have roles in dendritic growth and branching ( $\downarrow$ SEPT11, $\downarrow$ APOE, $\downarrow$ DNM1L, $\uparrow$ CTNNA2, $\uparrow$ MYO5A, $\uparrow$ RHOB, $\uparrow$ RTN4) and one of these RTN4 ( $\uparrow 1.76$ fold) a potent neurite outgrowth inhibitor (Chen et al., 2000). CTNNA2 ( $\uparrow 1.5$ fold) is a schizophrenia susceptibility gene (Chu and Liu, 2010), and functions in dendrite morphogenesis, cell adhesion, and regulation of synaptic structural plasticity and has recently been implicated in a pluripotent stem cell model of schizophrenia (Pedrosa et al., 2011). SEPT11 ( $\downarrow 1.6$ fold) has previously been observed to be reduced in the hippocampus in schizophrenia (Föcking et al., 2011). Importantly, six of the neuritogenesis proteins implicated here (SEPT11, RHOB, RALA, DNM1L, RTN4, MYO5A), have expression changes consistent with decreased neuritogenesis (Chen et al., 2000; Rao et al., 2002; Lalli and Hall, 2005; Li et al., 2009; McNair et al., 2010; Dickey and Strack, 2011), suggesting that neuritogenesis is reduced as a consequence of n-3 FA deficiency. These findings are in keeping with previous studies that show enhanced hippocampal neuritogenesis following DHA supplementation (Kawakita et al., 2006; Beltz et al., 2007; He et al., 2009).

Mitochondrial dysfunction is described in Alzheimer's, Huntington's, and Parkinson's disease (Eckert et al., 2013), and there is now increasing evidence implicating mitochondrial dysfunction in schizophrenia and mood disorders (Martins-De-Souza et al., 2011; Manji et al., 2012). Mitochondrial dysfunction occurs when the antioxidant defence system is overpowered by ROS-mediated oxidative stress, triggered by factors such as genetic defects, environmental exposures, and metabolic fluctuations. In the current study, we observed 10 mitochondrial dysfunction proteins to be significantly increased by n-3 FA deficiency ( $\uparrow$ ATP5A1, $\uparrow A T P 5 A B, \uparrow C O X 4 I 1, \uparrow N D U F A 8, \uparrow N D U F A 13, \uparrow N D U F V 1$, $\uparrow \mathrm{OGDH}, \uparrow \mathrm{PDHA} 1, \uparrow \mathrm{UQCRC} 1, \uparrow \mathrm{UQCR} 10)$. This uniform and significant up-regulation of mitochondrial dysfunction suggests n-3 FA deficiency is an environmental risk factor (dietary intake) capable of triggering cellular oxidative stress. In addition, mitochondria have important roles in regulating synaptic plasticity, learning, and memory, due to their key roles in processes such as ATP production (Manji et al., 2012), $\mathrm{Ca}^{2+}$ homeostasis (Gleichmann and Mattson, 2011), AMPA receptor trafficking in the synaptic membrane (Li et al., 2010), and apoptosis (Youle and Strasser, 2008), the latter of which is particularly important during neurogenesis (Deng et al., 2010). Dysfunctions in these processes are important neuropathological features of neurodegenerative and psychiatric diseases, and indeed 7 of the 10 significant mitochondrial proteins have previously been implicated at the mRNA level in either Alzheimer's, Huntington disease, or schizophrenia (Supplementary Table S1). In addition, ATP5A1 has previously been identified as significantly increased in proteomic studies of post-mortem brain in schizophrenia (English et al., 2011) and bipolar disorder (Pennington et al., 2008). In support of these findings, in vivo and ex vivo animal studies showed an improvement in mitochondrial function after treatment with n-3 FA (Eckert et al., 2013); and more specifically augmentation with DHA improved mitochondrial ATP generation in the CA1 area of the hippocampus (Harbeby et al., 2012). Moreover, a recent study by Feng and colleagues found that maternal DHA supplementation protects against prenatal stress induced impairment of learning and memory, and normalized mitochondrial, apoptotic, and oxidative stress proteins altered as a consequence of prenatal stress (Feng et al., 2012). The ability of dietary n-3 FA to consistently and uniformly modulate mitochondrial function may thus be instrumental in improving cognitive and behavioral abnormalities, as well as providing neuroprotection in vulnerable diseases states. In addition, 14-3-3 signaling pathway was also increased by deficiency, with 7 proteins up-regulated ( $\uparrow$ PRKCE, $\uparrow$ TUBA1A, $\uparrow$ TUBA4A, $\uparrow$ GRB2, $\uparrow Y W H A E, \uparrow Y W H A Q, \uparrow Y W H A Z$ ). The 14-3-3 proteins are consistently implicated in schizophrenia proteomic studies (English et al., 2011), but not specifically (Zabel et al., 2008), and have a wide variety of functions including cell cycle control, cellular signaling, and stress response.

The expression of the 114 candidate proteins were examined following chronic treatment with the antipsychotic haloperidol in the hippocampus. Twenty-three of the 114 proteins were significantly altered by haloperidol treatment and of these 17 were differentially expressed in the opposite direction to that observed following n-3 FA deficiency. These included proteins involved in neuritogenesis ( $\downarrow$ CTNNA2, $\downarrow$ STMN1), mitochondrial dysfunction ( $\downarrow$ ATP5A1, $\downarrow$ ATP5B, $\downarrow$ UQCRC1), CME ( $\uparrow$ ARPCL5, $\downarrow$ HSPA8), ribosomal proteins ( $\uparrow$ RS18, $\uparrow$ RPS3A), and others (个ARL3, $\uparrow$ CASKIN1, $\downarrow$ GDI2, $\downarrow$ NSFL1C, $\downarrow$ PKM2, $\downarrow$ PRKACA, $\downarrow$ PYGB) as detailed in Supplementary Table $S 1$. These findings provide preliminary evidence that antipsychotic drug treatment and n-3 FA deficiency share differential expression of several protein, and this evidence provides clues as to the that pathways that may be implicated in disease pathology.

This study has limitations and we acknowledge that the major findings were not validated by traditional methods such as western blotting or ELISA. However, our quantification method was based on triplicate LC-MS/MS runs for each sample, a strategy that was not feasible in previous $2 \mathrm{D}$ gel based experiments. In addition, reliability and consistency was confirmed by visual inspection of spectra, trypsin autolysis peaks, and stable LCMS/MS retention times across runs (data not shown). Total peptide count (no. of peptides identified per protein) were checked for consistency within replicate runs, and across samples to ensure the quality of the data. It should also be noted that our model of deficiency, through 2 generations, maybe considered extreme but previous work in these animals had demonstrated greater learning and cogitative defects in the second generation, as well as alterations in dopamine availability, respectively (Moriguchi et al., 2000; Bondi et al., 2013).

This is the first study to extensively characterize the proteomic and molecular pathways disturbed in the hippocampus as consequence of n-3 FA deficiency. In light of this data, it is conceivable that abnormalities of synaptic function, neuritogenesis, and mitochondrial dysfunction exists in individuals chronically 
deficient in n-3 FA, and that the modulation of these processes are a mechanisms by which n-3 FA improve symptoms in subjects in the at risk mental state (Amminger et al., 2010). This suggestion is in keeping with findings that neuritogenesis (Kawakita et al., 2006; Beltz et al., 2007; He et al., 2009) and mitochondrial function (Eckert et al., 2013) are significantly improved following n-3 augmentation, including that following prenatal stress (Feng et al., 2012), and these changes coincided with improvements in cognitive and depressive-like behaviors. Future studies will focus on understanding the brain proteomic response to n-3 FA supplementation following deficiency, and indeed by studying sub-proteomes such as membrane proteins (English et al., 2012) in n-3 deficient or $n-3$ ameliorated states.

\section{ACKNOWLEDGMENTS}

We gratefully acknowledge Professor Alan Mackay-Sims, Griffith University, Australia, for access to Ingenuity Pathway Analysis, and we are indebted to Dr Norman Salem, DSM Nutritional Lipids, U.S.A., for initiating our fruitful collaboration with Toru Moriguchi. Jane A. English and David R. Cotter are funded by the Health Research Board/Clinical Scientist Award. Melanie Föcking is funded by NARSAD, and Gerard Cagney is funded by Science Foundation Ireland.

\section{REFERENCES}

Allen, N. C., Bagade, S., McQueen, M. B., Ioannidis, J. P., Kavvoura, F. K., Khoury, M. J., et al. (2008). Systematic meta-analyses and field synopsis of genetic association studies in schizophrenia: the SzGene database. Nat. Genet. 40, 827-834. doi: 10.1038/ng.171

Amminger, G. P., Schafer, M. R., Papageorgiou, K., Klier, C. M., Cotton, S. M., Harrigan, S. M., et al. (2010). Long-chain omega-3 fatty acids for indicated prevention of psychotic disorders: a randomized, placebo-controlled trial. Arch. Gen. Psychiatry 67, 146-154. doi: 10.1001/archgenpsychiatry.2009.192

Beltz, B. S., Tlusty, M. F., Benton, J. L., and Sandeman, D. C. (2007). Omega-3 fatty acids upregulate adult neurogenesis. Neurosci. Lett. 415, 154-158. doi: 10.1016/j.neulet.2007.01.010

Ben Gedalya, T., Loeb, V., Israeli, E., Altschuler, Y., Selkoe, D. J., and Sharon, R. (2009). Alphasynuclein and polyunsaturated fatty acids promote clathrin-mediated endocytosis and synaptic vesicle recycling. Traffic 10, 218-234. doi: 10.1111/j.1600-0854.2008.00853.x

Bondi, C. O., Taha, A. Y., Tock, J. L., Totah, N. K., Cheon, Y., Torres, G. E., et al. (2013). Adolescent behavior and dopamine availability are uniquely sensitive to dietary omega-3 fatty acid deficiency. Biol. Psychiatry. doi: 10.1016/j.biopsych. 2013.06.007. [Epub ahead of print].

Chen, M. S., Huber, A. B., Van Der Haar, M. E., Frank, M., Schnell, L., Spillmann, A. A., et al. (2000). Nogo-A is a myelin-associated neurite outgrowth inhibitor and an antigen for monoclonal antibody IN-1. Nature 403, 434-439. doi: $10.1038 / 35000219$

Chu, T. T., and Liu, Y. (2010). An integrated genomic analysis of gene-function correlation on schizophrenia susceptibility genes. J. Hum. Genet. 55, 285-292. doi: 10.1038/jhg.2010.24

Deng, W., Aimone, J. B., and Gage, F. H. (2010). New neurons and new memories: how does adult hippocampal neurogenesis affect learning and memory. Nat. Rev. Neurosci. 11, 339-350. doi: 10.1038/nrn2822

Dickey, A. S., and Strack, S. (2011). PKA/AKAP1 and PP2A/Bbeta2 regulate neuronal morphogenesis via Drp1 phosphorylation and mitochondrial bioenergetics. J. Neurosci. 31, 15716-15726. doi: 10.1523/JNEUROSCI.315911.2011

Eckert, G. P., Lipka, U., and Muller, W. E. (2013). Omega-3 fatty acids in neurodegenerative diseases: focus on mitochondria. Prostaglandins Leukot. Essent. Fatty Acids 88, 105-114. doi: 10.1016/j.plefa.2012.05.006

\section{SUPPLEMENTARY MATERIAL}

The Supplementary Material for this article can be found online at: http://www.frontiersin.org/journal/10.3389/fgene.2013.00208/ abstract

\section{Supplementary Table S1 | List of 114 proteins significantly differentially} expressed as a consequence of dietary $n-3$ deficiency in the

mouse hippocampus. The table is ordered $p$-value, and proteins that were significant after permutation based FDR at $5 \%$ threshold are marked with an*. Further details on fold change, protein name, and the fold change of proteins that were significant in the haloperidol treated mouse hippocampus are also provided for each protein entry. In addition, IPA information on the most significant canonical pathways and neurological diseases are included.

Supplementary Figure S1 | (A) Representative MS1 peak which eluted at 12.75 minutes, with a $\mathrm{m} / \mathrm{z}$ of 628.82 . The peak width ranged between 12.41 and $12.92 \mathrm{~min}$ with at least 15 data points acquired across the $30 \mathrm{~s}$ peak. (B) Representative MS1 peak which eluted at $19.93 \mathrm{~min}$, with a $\mathrm{m} / \mathrm{z}$ of 360.72 . The peak width ranged between 19.76 and 20.30 min with at least14 data points acquired across the $30 \mathrm{~s}$ peak. (C) Representative MS1 peak which eluted at $22.86 \mathrm{~min}$, with a $\mathrm{m} / \mathrm{z}$ of 528.26 . The peak width ranged between 22.56 and 23.13 min with at least 23 data points acquired across the $30 \mathrm{~s}$ peak.

\section{Supplementary Figure S2 | The top IPA biological functions disrupted as a} consequence of $n-3$ FA deficiency.

English, J. A., Pennington, K., Dunn, M. J., and Cotter, D. R. (2011). The neuroproteomics of schizophrenia. Biol. Psychiatry 69, 163-172. doi: 10.1016/j.biopsych.2010.06.031

English, J. A., Manadas, B., Cotter, D. R., and Dunn, M. J. (2012). Partitioning the proteome: phase separation for targeted analysis of membrane proteins in human post-mortem brain. PLoS ONE 7:e39509. doi: 10.1371/journal. pone.0039509

Feng, Z., Zou, X., Jia, H., Li, X., Zhu, Z., Liu, X., et al. (2012). Maternal docosahexaenoic acid feeding protects against impairment of learning and memory and oxidative stress in prenatally stressed rats: possible role of neuronal mitochondria metabolism. Antioxid. Redox Signal. 16, 275-289. doi: 10.1089/ars.2010.3750

Ferraz, A. C., Delattre, A. M., Almendra, R. G., Sonagli, M., Borges, C., Araujo, P., et al. (2011). Chronic omega-3 fatty acids supplementation promotes beneficial effects on anxiety, cognitive and depressive-like behaviors in rats subjected to a restraint stress protocol. Behav. Brain Res. 219, 116-122. doi: 10.1016/j.bbr.2010.12.028

Föcking, M., Dicker, P., English, J. A., Cagney, G., and Cotter, D. R. (2012). Proteomic investigation of the postsynaptic density in the brain in schizophrenia. Schizophr. Res. 136,
S233. doi: $10.1016 /$ S0920-9964(12) 70704-1

Föcking, M., Dicker, P., English, J. A., Schubert, K. O., Dunn, M. J., and Cotter, D. R. (2011). Common proteomic changes in the hippocampus in schizophrenia and bipolar disorder and particular evidence for involvement of cornu ammonis regions 2 and 3 . Arch. Gen. Psychiatry 68, 477-488. doi: 10.1001/archgenpsychiatry. 2011.43

Gauthier, J., Champagne, N., Lafreniere, R. G., Xiong, L., Spiegelman, D., Brustein, E., et al. (2010). De novo mutations in the gene encoding the synaptic scaffolding protein SHANK3 in patients ascertained for schizophrenia. Proc. Natl. Acad. Sci. U.S.A. 107, 7863-7868. doi: $10.1073 /$ pnas.0906232107

Gleichmann, M., and Mattson, M. P. (2011). Neuronal calcium homeostasis and dysregulation. Antioxid. Redox Signal. 14, 1261-1273. doi: 10.1089/ars.2010.3386

Harauma, A., and Moriguchi, T. (2011). Dietary n-3 fatty acid deficiency in mice enhances anxiety induced by chronic mild stress. Lipids 46, 409-416. doi: 10.1007/s11745-0103523-z

Harbeby, E., Jouin, M., Alessandri, J. M., Lallemand, M. S., Linard, A., Lavialle, M., et al. (2012). n3 PUFA status affects expression 
of genes involved in neuroenergetics differently in the frontoparietal cortex compared to the CA1 area of the hippocampus: effect of rest and neuronal activation in the rat. Prostaglandins Leukot. Essent. Fatty Acids 86, 211-220. doi: 10.1016/j.plefa.2012.04.008

Harrison, P. J., and Weinberger, D. R. (2005). Schizophrenia genes, gene expression, and neuropathology: on the matter of their convergence. Mol. Psychiatry 10, 40-68. image 45. doi: 10.1038/sj.mp. 4001558

He, C., Qu, X., Cui, L., Wang, J., and Kang, J. X. (2009). Improved spatial learning performance of fat-1 mice is associated with enhanced neurogenesis and neuritogenesis by docosahexaenoic acid. Proc. Natl. Acad. Sci. U.S.A. 106, 11370-11375. doi: 10.1073/pnas.0904835106

Horrobin, D. F. (1998). The membrane phospholipid hypothesis as a biochemical basis for the neurodevelopmental concept of schizophrenia. Schizophr. Res. 30, 193-208. doi: 10.1016/S0920-9964(97)00151-5

Hubner, N. C., Bird, A. W., Cox, J., Splettstoesser, B., Bandilla, P., Poser, I., et al. (2010). Quantitative proteomics combined with BAC TransgeneOmics reveals in vivo protein interactions. J. Cell Biol. 189, 739-754. doi: 10.1083/jcb.200 911091

Kawakita, E., Hashimoto, M., and Shido, O. (2006). Docosahexaenoic acid promotes neurogenesis in vitro and in vivo. Neuroscience 139, 991-997. doi: 10.1016/j.neuro science.2006.01.021

Kim, J. Y., Liu, C. Y., Zhang, F., Duan, X., Wen, Z., Song, J., et al. (2012). Interplay between DISC1 and GABA signaling regulates neurogenesis in mice and risk for schizophrenia. Cell 148, 1051-1064. doi: 10.1016/j.cell.2011. 12.037

Kitajka, K., Puskas, L. G., Zvara, A., Hackler, L. Jr., Barcelo-Coblijn, G., Yeo, Y.K. et al. (2002). The role of n-3 polyunsaturated fatty acids in brain: modulation of rat brain gene expression by dietary n-3 fatty acids. Proc. Natl. Acad. Sci. U.S.A. 99, 2619-2624. doi: $10.1073 /$ pnas.042698699
Kuperstein, F., Yakubov, E., Dinerman, P., Gil, S., Eylam, R., Salem, N. Jr., et al. (2005). Overexpression of dopamine receptor genes and their products in the postnatal rat brain following maternal n-3 fatty acid dietary deficiency. J. Neurochem. 95, 1550-1562. doi: 10.1111/j.14714159.2005.03513.x

Lalli, G., and Hall, A. (2005). Ral GTPases regulate neurite branching through GAP-43 and the exocyst complex. J. Cell Biol. 171, 857-869. doi: $10.1083 /$ jcb. 200507061

Lepage, G., and Roy, C. C. (1986). Direct transesterification of all classes of lipids in a one-step reaction. J. Lipid Res. 27, 114-120.

Li, X., Serwanski, D. R., Miralles, C. P., Nagata, K., and De Blas, A. L. (2009). Septin 11 is present in GABAergic synapses and plays a functional role in the cytoarchitecture of neurons and GABAergic synaptic connectivity. J. Biol. Chem. 284, 17253-17265. doi: 10.1074/jbc.M109.008870

Li, Z., Jo, J., Jia, J. M., Lo, S. C., Whitcomb, D. J., Jiao, S., et al. (2010). Caspase-3 activation via mitochondria is required for long-term depression and AMPA receptor internalization. Cell 141, 859-871. doi: 10.1016/j.cell.2010.03.053

Manji, H., Kato, T., Di Prospero, N. A., Ness, S., Beal, M. F., Krams, M., et al. (2012). Impaired mitochondrial function in psychiatric disorders. Nat. Rev. Neurosci. 13, 293-307. doi: 10.1038/nrn3229

Martins-De-Souza, D., Harris, L. W., Guest, P. C., and Bahn, S. (2011). The role of energy metabolism dysfunction and oxidative stress in schizophrenia revealed by proteomics. Antioxid. Redox Signal. 15, 2067-2079. doi: 10.1089/ars.2010.3459

McNair, K., Spike, R., Guilding, C., Prendergast, G. C., Stone, T. W., Cobb, S. R., et al. (2010). A role for RhoB in synaptic plasticity and the regulation of neuronal morphology. J. Neurosci. 30, 3508-3517. doi: 10.1523/JNEUROSCI.538609.2010

McNamara, R. K. (2009). Evaluation of docosahexaenoic acid deficiency as a preventable risk factor for recurrent affective disorders: current status, future directions, and dietary recommendations. Prostaglandins Leukot. Essent. Fatty Acids 81, 223-231. doi: 10.1016/j.plefa.2009.05.017

McNamara, R. K. (2011). Omega3 fatty acid deficiency: a preventable risk factor for schizophrenia. Schizophr. Res. 129, 215-216. doi: 10.1016/j.schres.2010.12.017

Moriguchi, T., Greiner, R. S., and Salem, N., Jr. (2000). Behavioral deficits associated with dietary induction of decreased brain docosahexaenoic acid concentration. J. Neurochem. 75, 2563-2573. doi: 10.1046/j.14714159.2000.0752563.x

Ozyurt, B., Sarsilmaz, M., Akpolat, N., Ozyurt, H., Akyol, O., Herken, H., et al. (2007). The protective effects of omega- 3 fatty acids against MK-801-induced neurotoxicity in prefrontal cortex of rat. Neurochem. Int. 50, 196-202. doi: 10.1016/j.neuint.2006.08.002

Pedrosa, E., Sandler, V., Shah, A., Carroll, R., Chang, C., Rockowitz, S., et al. (2011). Development of patient-specific neurons in schizophrenia using induced pluripotent stem cells. J. Neurogenet. 25, 88-103. doi: 10.3109/01677063.2011.597908

Pennington, K., Beasley, C. L., Dicker, P., Fagan, A., English, J., Pariante, C. M., et al. (2008). Prominent synaptic and metabolic abnormalities revealed by proteomic analysis of the dorsolateral prefrontal cortex in schizophrenia and bipolar disorder. Mol. Psychiatry 13, 1102-1117. doi: 10.1038/sj.mp.400 2098

Rao, M. V., Engle, L. J., Mohan, P. S., Yuan, A., Qiu, D., Cataldo, A., et al. (2002). Myosin Va binding to neurofilaments is essential for correct myosin $\mathrm{Va}$ distribution and transport and neurofilament density. J. Cell Biol. 159, 279-290. doi: $10.1083 /$ jcb. 20020 5062

Reeves, P. G., Nielsen, F. H., and Fahey, G. C., Jr. (1993). AIN-93 purified diets for laboratory rodents: final report of the American Institute of Nutrition ad hoc writing committee on the reformulation of the
AIN-76A rodent diet. J. Nutr. 123, 1939-1951.

Schubert, K. O., Focking, M., Prehn, J. H., and Cotter, D. R. (2012). Hypothesis review: are clathrin-mediated endocytosis and clathrin-dependent membrane and protein trafficking core pathophysiological processes in schizophrenia and bipolar disorder. Mol. Psychiatry 17, 669-681. doi: 10.1038/mp.2011.123

Youle, R. J., and Strasser, A. (2008). The BCL-2 protein family: opposing activities that mediate cell death. Nat. Rev. Mol. Cell Biol. 9, 47-59. doi: 10.1038/nrm2308

Zabel, C., Andreew, A., Mao, L., and Hartl, D. (2008). Protein expression overlap: more important than which proteins change in expression. Expert Rev. Proteomics 5, 187-205. doi: 10.1586/14789450.5. 2.187

Conflict of Interest Statement: The authors declare that the research was conducted in the absence of any commercial or financial relationships that could be construed as a potential conflict of interest.

Received: 30 July 2013; accepted: 30 September 2013; published online: 28 October 2013.

Citation: English JA, Harauma A, Föcking M, Wynne K, Scaife C, Cagney G, Moriguchi T and Cotter DR (2013) Omega-3 fatty acid deficiency disrupts endocytosis, neuritogenesis, and mitochondrial protein pathways in the mouse hippocampus. Front. Genet. 4:208. doi: 10.3389/fgene. 2013.00208

This article was submitted to Neurogenomics, a section of the journal Frontiers in Genetics.

Copyright (c) 2013 English, Harauma, Föcking, Wynne, Scaife, Cagney, Moriguchi and Cotter. This is an openaccess article distributed under the terms of the Creative Commons Attribution License (CC BY). The use, distribution or reproduction in other forums is permitted, provided the original author(s) or licensor are credited and that the original publication in this journal is cited, in accordance with accepted academic practice. No use, distribution or reproduction is permitted which does not comply with these terms. 